\title{
DURATION AND TIMING OF SPAWNING SEASONS IN MARINE TELEOSTS: A BIOGEOGRAPHICAL APPROACH
}

\author{
Fayakun Satria")
}

\begin{abstract}
The duration and timing patterns of spawning seasons among marine teleosts fishes over broad geographic ranges from the polars to the equator has been reviewed. The review was based on 206 studies in order to evaluate potential patterns of the length or timing of spawning seasons in relation to latitude, habitats and taxonomic groupings patterns. This review confirmed that the timing and duration of spawning of marine teleost fishes is related to latitude. The spawning duration of fishes living in the equatorial region and the tropical regions is generally longer than that of species living in the subtropical and polar regions. With respect to their habitat, in general, the duration and timing patterns of spawning seasons of pelagic fish were not different to demersal fishes.
\end{abstract}

KEYWORDS: spawn, seasons, duration, marine teleost fish

\section{INTRODUCTION}

The duration and timing of spawning seasons vary substantially among marine teleost fishes (Taylor, 1990; Munro et al., 1990). Breeding seasons can range from periods of just a few days (Bye, 1990; Beddow et al., 1998) to all year round (Stequert \& Ramcharrun, 1996). Among the seasonal breeders, there is also considerable variation in the time of the year when breeding occurs (Sundararaj, 1981; Longhurst \& Pauly, 1987). Some species spawn mainly during summer (Sabates \& Martin, 1993), while other spawn during winter (Amara et al., 1994; (Fowler et al., 1999). The processes underlying variation in the duration and timing of spawning seasons are poorly understood. To date there has been no comprehensive review of patterns in the length or timing of spawning seasons, making it difficult to formulate appropriate theories.

The timing and duration of spawning seasons appear to vary with latitude, both within and among species (Taylor, 1990). It is widely assumed that the length of the spawning season increases toward the equator as temperature increases and becomes less variable (Munro et al., 1990). However, there have been no systematic comparisons over a broad latitudinal range to confirm this. Most studies on the spawning seasons of marine fish have been restricted to a single locality and also at high latitude locations. This restricts our ability to determine how single species alter their spawning patterns over potentially broad geographic ranges. However, over recent years, information on the spawning patterns of tropical species has been increasing. There have now been sufficient studies on a range of species from different locations to detect major trends from the polar regions to equatorial environments.

There are several factors that are widely assumed as potential causative factors of the timing of the reproductive season. Photoperiod, temperature, rainfall and food, among other factors are important in regulating reproductive cycles in teleost fishes (Baggerman, 1990; Taylor, 1990). Many species live over a wide range of latitudes and so encounter different temperature and photoperiod regimes at different locations. This can result in major differences in the timing and duration of reproduction within species (Wootton, 1990). Reproductive seasonality has been well described for high latitude species and is correlated with major seasonal changes in temperature and hours of daylight (Wootton, 1990). Temperature and photoperiod appear to influence both the timing and duration of the spawning season, with most temperate species having restricted spawning periods (Munro et al., 1990). However, strong reproductive seasonality has also been observed at low latitudes in some freshwater and marine species (Bye, 1990; Taylor, 1990). This suggests that the assumption of longer spawning seasons in the tropics may not always apply. Before any conclusions can be drawn, however, a systematic comparison of spawning seasons of different taxa across a wide range of latitudes is necessary.

Marine teleost fishes living in different habitats (e.g. demersal and pelagic fish) experience different abiotic and biotic conditions. Micro climates associated with habitats may also influence reproductive strategies, including

\footnotetext{
Research Center for Capture Fisheries, Muara Baru-Jakarta
} 
breeding at a time and place which is most conducive to the survival of their offspring (Bye, 1990). It is thought that pelagic fishes tend to have longer spawning periods than demersal fish. For example the dolphin fish, Coryhaena hippuru (Wu et al., 2001) and the skipjack tuna, Katsuwonus pelamis (Stequert \& Ramcharrun, 1996) spawn all year around, while a demersal fish, the Black grouper, Mcyertoperca bonaci, spawns over a six month period (Crabtree \& Bullock L., 1998). Pelagic fishes are highly mobile and are able to migrate to areas where conditions are optimal for their growth and reproduction. For example, they are able to move to areas where food availability is high, and to warmer areas during winter. In contrast most demersal species are confined to a particular area where local conditions may not always be favourable for reproduction. This was explained why pelagic species are likely to have a longer spawning period than demersal fish. However there are no studies comparing the reproductive timing of fishes living in different habitats over a large geographical scale to help distinguish between the effects of habitat and latitude on the marine teleosts.

The purpose of this review is to draw together information from disparate sources to examine variation in the timing and duration of spawning seasons in marine fishes. The review will evaluate potential patterns in relation to latitude, habitats and taxonomic groupings. The following specific questions will be addressed:

1. Is there a trend toward longer spawning seasons both within and among species from high to low latitude?

2. Is there systematic variation in the timing of spawning both within and among species in relation to latitude?

3. Is there a consistent difference between demersal and pelagic fishes in the duration of spawning seasons?

\section{PATTERNS IN THE DURATION OF SPAWNING PERIOD IN MARINE TELEOSTS WITH RESPECT TO LATITUDINAL RANGE}

A through investigation of reproductive studies on marine teleosts from a variety of latitudinal ranges will help us to gain a better understanding on the spawning patterns of marine teleosts across different latitudinal ranges. To examine the relationship between the duration of the spawning season and latitude, I have drawn on 206 studies which mostly conducted between 1993 to 2002 (Table 1).

Table 1. Summary of the duration and timing of spawning seasons in marine teleosts from the polar towards equatorial regions

\begin{tabular}{lclll}
\hline \multicolumn{1}{c}{ Species } & Month & Spawning season & Latitude & \multicolumn{1}{c}{ References } \\
\hline Acanthopagrus berda & 4 & Jun-Sept & $17.00 \mathrm{~S}$ & (Sheaves et al., 1999) \\
Acanthopagrus schlegeli & 4 & Jan-Apr & $24.00 \mathrm{~N}$ & (Huang \& Chiu, 1998) \\
Acanthurus lineatus & 5 & Oct-Feb & $14.00 \mathrm{~S}$ & (Craig et al., 1997) \\
Achoerodus Viridis & 4 & Jul-Oct & $33.58 \mathrm{~S}$ & (Gillanders, 1995) \\
Albula vulpes & 7 & Nov-May & $13.11 \mathrm{~N}$ & (Crabtree et al., 1997b) \\
Alepochephalus bairdii & 4 & Jan-Apr & $55.00 \mathrm{~N}$ & (Allain, 2001) \\
Amblyglyphidodon leugaster & 5 & May-Sept & $29.30 \mathrm{~N}$ & (Goulet, 1995) \\
Ammodytes hexapterus & 2 & Sept-Oct & $59.17 \mathrm{~N}$ & (Robards et al., 1999) \\
Ammodytes marinus & 2 & Des-Jan & $61.00 \mathrm{~N}$ & (Bergstad, O. A, 2001) \\
Anguilla japonica & 3 & May-Jul & $16.00 \mathrm{~N}$ & (Ishikawa et al., 2001) \\
Aphanius iberus & 7 & Mar-Sept & $41.00 \mathrm{~N}$ & (Vargas \& Desostoa, 1996) \\
Apogon lineatus & 4 & Jul-Oct & $35.00 \mathrm{~N}$ & (Kume et al., 2000) \\
Apogon ngripinnis & 6 & Oct-Mar & $26.10 \mathrm{~S}$ & (Almeida et al., 1999) \\
Arripis georgiana & 2 & May-Jun & $2843 \mathrm{~S}$ & (Fairclough et al., 2000) \\
Atherina boyeri & 8 & Feb-Sept & $43.10 \mathrm{~N}$ & (Tomasini et al., 1996) \\
Atherinomorus lacunosus & 5 & Aug-Dec & $21.00 \mathrm{~S}$ & (Conand, 1993) \\
Athrobucca nibe & 8 & Sept-Feb/Aug/Mar & $30.00 \mathrm{~S}$ & (Fennessy, 2000) \\
Beryx splendens & 4 & Nov-Feb & $20.15 \mathrm{~S}$ & (Lehodey et al., 1997) \\
Boreogadus saida & 5 & Aug-Nov/Feb & $80.00 \mathrm{~N}$ & (Hop et al., 1995) \\
Brevoortia aurea & 5 & Sept-Jan & $35.00 \mathrm{~S}$ & (Acha \& Macchi, 2000) \\
Brevoortia tyrannus & 2 & Dec-Jan & $34.36 \mathrm{~N}$ & (Warlen, 1994) \\
\hline
\end{tabular}


Table 1. Countinous

\begin{tabular}{|c|c|c|c|c|}
\hline Species & Month & Spawning season & Latitude & References \\
\hline Capoeta capoeta umbla & 3 & Mar-Jul & $39.56 \mathrm{~N}$ & (Turkmen et al., 2001) \\
\hline Caranx bucculentus & 2 & Aug-Sept & $14.00 \mathrm{~N}$ & (Brewer et al., 1994) \\
\hline Caulolatilus princeps & 5 & Jan-Apr/Aug-Sept & $24.45 \mathrm{~N}$ & (Garay \& Luna, 1994) \\
\hline Centropomus undecimalis & 5 & Apr-Aug & $27.45 \mathrm{~N}$ & (Peters et al., 1998) \\
\hline Centropomus undecimalis & 5 & May-Sept & $27.30 \mathrm{~N}$ & (Taylor et al., 1998) \\
\hline Centropristis striata & 5 & Dec-Apr & $28.10 \mathrm{~N}$ & (Hood et al., 1994) \\
\hline Centropumus undecimalis & 7 & Apr-Oct & $28.00 \mathrm{~N}$ & (Taylor et al., 1998) \\
\hline Centroscyllium fabricii & 1 & Oct & $65.00 \mathrm{~S}$ & (Jacobsdottir, 2001) \\
\hline Cepola rubescens & 6 & Mar-Oct & $39.12 \mathrm{~N}$ & (Stergiou et al., 1996) \\
\hline Chelidonichthys kumu & 7 & Sept-Mar & $34,00 \mathrm{~S}$ & (Clearwater \& Pankhurst, 1994) \\
\hline Chionodraco hamatus & 3 & Dec-Feb & $60.00 \mathrm{~S}$ & (Vacchi et al., 1996) \\
\hline Chionodraco hamatus & 3 & Jul-Sept & $68.30 \mathrm{~S}$ & (Vacchi et al., 1996) \\
\hline Choerodon schoenlenii & 4 & Feb-May & $26.17 \mathrm{~N}$ & (Ebisawa et al., 1995) \\
\hline Chrypsitera rolandi & 12 & Jan-Dec & $05.00 \mathrm{~N}$ & (Srinivasan et al., 2002) \\
\hline Clupea harengus & 1 & Feb-mar & $64.00 \mathrm{~N}$ & (Slotte \& Fiksen, 2000) \\
\hline Clupea harengus & 2 & Feb-mar & $64.00 \mathrm{~N}$ & (Slotte et al., 2000) \\
\hline Clupea harengus & 5 & Sept-Jan & $54.30 \mathrm{~N}$ & (Dickey-Collas et al., 2001) \\
\hline Clupea harengus membras & 4 & Apr-Jul & $60.00 \mathrm{~N}$ & (Rajasilta et al., 2001) \\
\hline Clupea harengus membras & 5 & Apr-Aug & $57.00 \mathrm{~N}$ & (Anderson et al., 2000) \\
\hline Clupeoid ophistomena & 7 & Jan-Mar/Jul-Sept & $31.00 \mathrm{~N}$ & (Acal \& Corroespinosa, 1994) \\
\hline Cnidoglanis macrocephalus & 3 & Oct-Jan & $33.30 \mathrm{~S}$ & (Laurenson et al., 1993) \\
\hline Coryhaena hippurus & 4 & Jul-Sept & $36.00 \mathrm{~N}$ & (Massuti \& Morales-Nin, 1995) \\
\hline Coryhaena hippurus L & 12 & Jan-Dec & $23.00 \mathrm{~N}$ & (Wu et al., 2001) \\
\hline Coryphaenoides rupestris & 5 & Jun-Nov & $56.00 \mathrm{~N}$ & (Kelly et al., 1996) \\
\hline Coryphaenoides rupestris & 10 & Feb-Nov & $55.00 \mathrm{~N}$ & (Allain, 2001) \\
\hline Cynoscion regalis & 4 & May-Aug & $37.30 \mathrm{~N}$ & (Barbieri et al., 1994) \\
\hline Dascyllus aruanus & 4 & Jun-Sept & $25.00 \mathrm{~N}$ & (Mizushima et al., 2000) \\
\hline Decapterus macrosoma & 6 & Jun-Nov & $05.00 \mathrm{~N}$ & $\begin{array}{l}\text { (Atmaja, S. B. \& B. Sadhotomo, } \\
\text { 2005) }\end{array}$ \\
\hline Decapterus russelli & 7 & Feb-Aug & $05.00 \mathrm{~N}$ & (Widodo, J., 1989) \\
\hline Dentex tumifrons & 8 & Sept-Des/Mar-Jun & $27.30 \mathrm{~N}$ & (Oki \& Tabeta, 1998) \\
\hline Dicentrarchus labrax & 2 & Feb-Mar & $40.00 \mathrm{~N}$ & (Mananos et al., 1997) \\
\hline Dicologlossa cuneata & 5 & Jan-May & $36.30 \mathrm{~N}$ & (Jimenez et al., 1998) \\
\hline Diplodus annularis & 5 & Jan-May & $29.00 \mathrm{~N}$ & (Pajuelo \& Lorenzo, 2001) \\
\hline Dosidicus gigas & 4 & Oct-Jan & $05.30 \mathrm{~S}$ & (Tafur et al., 2001) \\
\hline Dosidicus gigas & 2 & Jul-Aug & $14.30 \mathrm{~S}$ & (Tafur et al., 2001) \\
\hline Engraulis anchoita & 4 & Mar-Aug & $22.30 \mathrm{~S}$ & (Lima \& Castello, 1995) \\
\hline Engraulis encrasicolus & 6 & Mar-Aug & $46.00 \mathrm{~N}$ & (Motos et al., 1996) \\
\hline Engraulis encrasicolus & 2 & Jun-Jul & $43.30 \mathrm{~N}$ & (Nierrmann et al., 1994) \\
\hline Engraulis encrasicolus & 3 & Jul-Aug & $36.30 \mathrm{~N}$ & (Millan, 1999) \\
\hline Engraulis encrasicolus ponticus & 4 & May-Aug & $44.00 \mathrm{~N}$ & (Lisovenko \& Andrianov, 1996) \\
\hline Engraulis japonicus & 3 & Mar-Jun & $35.00 \mathrm{~N}$ & (Funamoto \& Aoki, 2001) \\
\hline Engraulis japonicus & 2 & Apr/Jun & $34.30 \mathrm{~N}$ & (Kim \& Nancy, 2001) \\
\hline Engraulis japonicus & 5 & Mar-May/Aug-Sept & $26.00 \mathrm{~N}$ & (Chiu \& Chen, 2001) \\
\hline Engraulis japonicus $S$ & 3 & Feb-Mar/Jul & $26.00 \mathrm{~N}$ & (Chiu \& Chen, 2001) \\
\hline Ephinephelus morio & 3 & Mar-May & $22.30 \mathrm{~N}$ & (Johnson et al., 1998) \\
\hline phinephelus niveatus & 6 & Apr-Sept & $33.30 \mathrm{~N}$ & (Wyanski et al., 2000) \\
\hline Ehinephelus polyphekadion & 2 & Mar-Jun & $36.20 \mathrm{~S}$ & (Rasem et al., 1997) \\
\hline pinephelus marginatus & 4 & Jul-Sept & $35.30 \mathrm{~N}$ & (Marino et al., 2001) \\
\hline pinephelus rivulatus & 6 & Jul-Dec & $22.00 \mathrm{~S}$ & (Mackie, 2000) \\
\hline
\end{tabular}


Table 1. Countinous

\begin{tabular}{|c|c|c|c|c|}
\hline Species & Month & Spawning season & Latitude & References \\
\hline Etmopterus princeps & 3 & Oct/Jun-Jul & $65.30 \mathrm{~S}$ & (Jacobsdottir, 2001) \\
\hline Gadus morhua & 3 & Jan-Mar & $52.30 \mathrm{~N}$ & (Brander, 1994) \\
\hline Gadus morhua & 3 & Apr-Jun & $57.30 \mathrm{~N}$ & (Anderson et al., 1995) \\
\hline Gadus morhua & 3 & Apr-Jun & $55.00 \mathrm{~N}$ & (Kai W. et al., 2000) \\
\hline Gadus morhua & 3 & Apr-Jun & $55.00 \mathrm{~N}$ & (Lawson \& Rose, 2000) \\
\hline Gadus morhua & 3 & Apr-Jun & $47.15 \mathrm{~N}$ & (Lawson \& Rose, 2000) \\
\hline Gambusia holbrooki & 5 & May-Sept & $41.00 \mathrm{~N}$ & (Vargas \& Desostoa, 1996) \\
\hline Gobius roulei & 5 & Apr-Aug & $45.16 \mathrm{~N}$ & $\begin{array}{l}\text { (Kovacic, 2001) } \\
\text { (Palazon-Fernandez et al., }\end{array}$ \\
\hline Halobatrachus didactylus & 6 & Mar-Aug & $36.32 \mathrm{~N}$ & 2001) \\
\hline Helicolenus dactylopterus & 4 & Mar-Jun & $55.00 \mathrm{~N}$ & (Allain, 2001) \\
\hline Helicolenus dactylopterus & 3 & Dec-Feb & $37.30 \mathrm{~N}$ & (Munoz et al., 1999) \\
\hline Hippoglossus hippoglossus & 3 & Jun, Nov-Dec & $44.00 \mathrm{~N}$ & (Neilson et al., 1993) \\
\hline Hirundichthys affinis & 7 & Dec-Jun & $13.10 \mathrm{~N}$ & (Khokiattiwong et al., 2000) \\
\hline Hucho perryi & 1 & Jul & $51.00 \mathrm{~N}$ & (Berg, L. S. et al, 1995) \\
\hline Hucho perryi & 1 & Apr & $47.30 \mathrm{~N}$ & (Fukushima, 1994) \\
\hline Hyperlophus vittatus & 5 & Mar-Sept & $32.55 \mathrm{~S}$ & (Gaughan et al., 1996) \\
\hline Hyperoglyphe antartica & 3 & Mar-May & $42.30 \mathrm{~S}$ & (Baelde, 1996) \\
\hline Hypomesus pretiosus japanicus & 3 & Mar-May & $37.30 \mathrm{~N}$ & (Hirose \& Kawaguchi, 1998) \\
\hline Johnius amblycephalus & 6 & Sept-Feb & $30.00 \mathrm{~S}$ & (Fennessy, 2000) \\
\hline Johnius dussumieri & 6 & Sept-Feb & $30.00 \mathrm{~S}$ & (Fennessy, 2000) \\
\hline Kaiwarinus equula & 6 & Mar-Oct & $30.00 \mathrm{~N}$ & (Yoneda et al., 2001a) \\
\hline Kareius bicoloratus & 1 & Nov & $35.00 \mathrm{~N}$ & (Uehara \& Shimizu, 1996) \\
\hline Katsuwonus pelamis & 12 & Jan-Dec & $20.00 \mathrm{~S}$ & (Stequert \& Ramcharrun, 1996) \\
\hline Lactophrys quadriconis & 6 & Jan-Feb/Jul-Sept & $10.40 \mathrm{~N}$ & (Ruiz et al., 1999) \\
\hline Lates calcarifer & 5 & Oct-Feb & $05.00 \mathrm{~N}$ & $\begin{array}{l}\text { (Guiguen et al., 1994) } \\
\text { (Jayawardane \& Dayaratne, }\end{array}$ \\
\hline Leiognathus brevirostris & 2 & Feb/Jul & $08.00 \mathrm{~N}$ & 1998) \\
\hline Limanda aspera & 4 & May-Aug & $60.05 \mathrm{~N}$ & (Paul et al., 1993) \\
\hline Lithognathus lithognathus & 2 & Mar-Apr & $33.00 \mathrm{~S}$ & (Bennett, 1993) \\
\hline Lophiomus setigerus & 7 & Mar-Nov & $30.00 \mathrm{~N}$ & (Yoneda et al., 1998) \\
\hline Lophius litulon & 4 & Feb-May & $32.30 \mathrm{~N}$ & (Yoneda et al., 2001b) \\
\hline Lutjanus campechanus & 2 & Mar-Jun & $42.13 \mathrm{~N}$ & (Soliman M. A. et al., 1992) \\
\hline Lutjanus fulviflamma & 6 & Dec-Apr & $02.00 \mathrm{~S}$ & (Kaundaarara \& Ntiba, 1997) \\
\hline Lutjanus vittus & 6 & Nov-Apr & $19.30 \mathrm{~N}$ & (Davis \& West, 1993) \\
\hline Megalops atlanticus & 4 & Apr-Aug & $23.35 \mathrm{~N}$ & (Crabtree et al., 1997a) \\
\hline Merluccius merluccius & 1 & Jun & $51.30 \mathrm{~N}$ & (Fives J. M. et al., 2001) \\
\hline Micromesistius poutassou & 1 & Apr & $51.30 \mathrm{~N}$ & (Fives J. M. et al., 2001) \\
\hline Micropogonias undulatus & 6 & Jun-Des & $37.30 \mathrm{~N}$ & (Barbieri et al., 1994) \\
\hline Microstomus pacificus & 6 & Dec-May & $45.00 \mathrm{~N}$ & (Crone, P. R., 2001) \\
\hline Mullus barbatus & 4 & Mar-Jun & $33.30 \mathrm{~N}$ & (Golani, 1994) \\
\hline Mycteroperca bonaci & 3 & Jan-Mar & $27.12 \mathrm{~N}$ & (Crabtree \& Bullock L., 1998) \\
\hline Mycteroperca bonaci & 5 & Jan-May & $21.30 \mathrm{~N}$ & $\begin{array}{l}\text { (Garciacagide \& Garcia, 1996) } \\
\text { (Bullock L. \& M. D. Murphy, }\end{array}$ \\
\hline Mycteroperca interstialis & 2 & Apr-May & $28.15 \mathrm{~N}$ & 1994) \\
\hline Mycteroperca venenosa & 5 & Jan-May & $21.30 \mathrm{~N}$ & (Garciacagide \& Garcia, 1996) \\
\hline Mysteroperca microlepis & 3 & Feb-Apr & $27.00 \mathrm{~N}$ & (Collins et al., 1998) \\
\hline Odontesthes bonariensis & 6 & Mar-May/Aug-Oct & $25.18 \mathrm{~S}$ & (Barros \& Regidor, 2002) \\
\hline Opisthonema oglinum & 4 & May-Aug & $21.00 \mathrm{~N}$ & (Garcia-Abad et al., 1998) \\
\hline Opisthonema oglinum & 6 & May-Oct & $19.30 \mathrm{~N}$ & (Garcia-Abad et al., 1998) \\
\hline Otolithes ruber & 6 & Sept-Feb & $30.00 \mathrm{~S}$ & (Fennessy, 2000) \\
\hline
\end{tabular}


Table 1. Countinous

\begin{tabular}{|c|c|c|c|c|}
\hline Species & Month & Spawning season & Latitude & References \\
\hline Pagellus acarne & 5 & May-Sept & $38.10 \mathrm{~N}$ & (Arculeo \& Brusle-Sicard, 2000) \\
\hline Pagellus acarne & 6 & Oct-Mar & $29.00 \mathrm{~N}$ & (Pajuelo \& Lorenzo, 2000) \\
\hline Pagrus pagrus & 4 & Jan-Apr & $25.00 \mathrm{~N}$ & (Hood \& Johnson, 2000) \\
\hline Pampus argenteus & 5 & Mar-May/Aug-Sept & $29.30 \mathrm{~N}$ & (Dadzie et al., 1998) \\
\hline Paralabrax maculatofsciatus & 3 & Jun-Aug & $39.00 \mathrm{~N}$ & (Allen et al., 1995) \\
\hline Parma microlepis & 5 & Aug-Jan & $34.00 \mathrm{~S}$ & (Fowler et al., 1999) \\
\hline Parma microlepis & 6 & Jul-Nov/Jan & $34.30 \mathrm{~S}$ & (Tzioumis \& Kingsford, 1999) \\
\hline Peprilus alepidotus & 2 & Jun-Jul & $37.00 \mathrm{~N}$ & (Rotunno \& Cowen, 1997) \\
\hline Peprilus burti & 6 & Feb-May/Sept-Nov & $37.00 \mathrm{~N}$ & (Rotunno \& Cowen, 1997) \\
\hline Peprilus triacanthus & 4 & May-Aug & $37.00 \mathrm{~N}$ & (Rotunno \& Cowen, 1997) \\
\hline Phocoena phocoena & 2 & Jul-Aug & $65.45 \mathrm{~N}$ & (Lockyer, C. et al., 2001) \\
\hline Platycephalus bassensis & 6 & Oct-Mar & $42.30 \mathrm{~S}$ & (Jordan, 2001) \\
\hline Plectropomus leopardus & 3 & Sept-Nov & $16.39 \mathrm{~S}$ & (Ferreira \& Russ, 1995) \\
\hline Pleuronectes platessa & 1 & Jan & $51.00 \mathrm{~N}$ & (Arnold \& Metcalfe, 1996) \\
\hline Pleuronectes platessa & 3 & Feb-Apr & $54.00 \mathrm{~N}$ & (Ellis \& Nash, 1997) \\
\hline Pomatomus saltatrix & 2 & Apr-May & $45.00 \mathrm{~N}$ & (Crone, P. R., 1994) \\
\hline Pomatomus saltatrix & 3 & Jul-Sept & $41.15 \mathrm{~N}$ & (Sabates \& Martin, 1993) \\
\hline Priacanthus macracanthus & 2 & May-Jun & $27.30 \mathrm{~N}$ & (Oki \& Tabeta, 1999) \\
\hline Priacanthus macracanthus & 4 & Apr-Jul & $24.45 \mathrm{~N}$ & (Liu et al., 2001) \\
\hline Psetta maxima & 3 & Mar-May & $42.30 \mathrm{~N}$ & (Caputo et al., 2001) \\
\hline Pseudolabrus celidotus & 5 & Jul-Nov & $38.15 \mathrm{~S}$ & (Jones, 1980) \\
\hline Pseudopercis semifasciata & 4 & Oct-Jan & $47.00 \mathrm{~S}$ & (Macchi et al., 1995) \\
\hline Rastrelliger kanagurta & 6 & Mar-Aug & $05.00 \mathrm{~N}$ & (Nurhakim, S., 1993) \\
\hline Reinhardtius hippoglossoides & 3 & Nov-Jan & $73.00 \mathrm{~N}$ & (Albert et al., 2001) \\
\hline Repomucenus valenciennei & 8 & Apr-Nov & $35.00 \mathrm{~N}$ & (Ikejima \& Shimizu, 1999) \\
\hline Rhincodon typus & 2 & Apr-May & $16.30 \mathrm{~N}$ & (Heyman et al., 2001) \\
\hline Rhomboplites aurorubens & 3 & Jun-Aug & $32.00 \mathrm{~N}$ & (Zhao et al., 1997) \\
\hline Rhomboplites aurorubens & 8 & Apr-Nov & $30.00 \mathrm{~N}$ & (Cuellar et al., 1996) \\
\hline Rhomboplites aurorubens & 5 & Mar-Sept & $25.00 \mathrm{~N}$ & (Hood \& Johnson, 1999) \\
\hline Salvelinus alpinus & 1 & Sept & $58.00 \mathrm{~N}$ & (Beddow et al., 1998). \\
\hline Sardinella aurita & 6 & Jun-Oct/Dec/Mar & $04.00 \mathrm{~N}$ & (Quaatey \& Maravelias, 1999) \\
\hline Sardinella maderensis & 3 & Jan/Apr-May & $02.55 \mathrm{~N}$ & $\begin{array}{l}\text { (Gabche \& Hockey, 1995) } \\
\text { (Quinonez-Velaquez et al., }\end{array}$ \\
\hline Sardinops caeruleus & 7 & Oct-Apr & $28.00 \mathrm{~N}$ & 2000) \\
\hline Sardinops melanostictus & 1.5 & Feb-Mar & $32.00 \mathrm{~N}$ & (Aoki \& Murayama, 1993) \\
\hline Sarotherodon melanotheron & 2 & Apr-May & $28.00 \mathrm{~N}$ & (Faunce, 2000) \\
\hline sarpa salpa & 6 & Apr-Sept & $30.54 \mathrm{~N}$ & (Van Der Walt \& B. Q., 1998) \\
\hline Sciaenops ocellatus & 3 & Jul-Sept & $35.10 \mathrm{~N}$ & (Ross et al., 1995) \\
\hline Scomber scombrus & 2 & Jun-Jul & $51.30 \mathrm{~N}$ & (Fives J. M. et al., 1994) \\
\hline Scomberomus commerson & 2 & Oct-Nov & $19.00 \mathrm{~S}$ & (Mcpherson, 1993) \\
\hline Scophthalmus rombus & 7 & Jan-Jul & $42.30 \mathrm{~N}$ & (Caputo et al., 2001) \\
\hline Sebastolobus macrochir & 2 & Mar-Apr & $45.00 \mathrm{~N}$ & (Koya et al., 1995) \\
\hline Selaroides leptolepis & 4 & Sept-Oct/Jan-Feb & $17.30 \mathrm{~N}$ & (Venkataramani et al., 1995) \\
\hline Seranus cabrilla & 6 & Feb-Jul & $29.00 \mathrm{~N}$ & (Garcia-Diaz et al., 1997) \\
\hline Seriola laladi & 1 & Des & $37.30 \mathrm{~S}$ & (Gillanders 'et al., 1999) \\
\hline Seriolella brama & 4 & May-Aug & $40.00 \mathrm{~S}$ & (Knuckey \& Sivakumaran, 2001) \\
\hline Serranus cabrilla & 6 & Feb-Jul & $28.00 \mathrm{~N}$ & (Garcia-Diaz et al., 1997) \\
\hline Siganus spinus & 3 & May-Dec & $26.30 \mathrm{~N}$ & (Harahap et al., 2001) \\
\hline Siganus sutor & 6 & Sept-Feb & $26.10 \mathrm{~S}$ & (Almeida et al., 1999) \\
\hline Sillaginodes punctata & 4 & Jun-Sept & $32.20 \mathrm{~S}$ & (Hyndes et al., 1998) \\
\hline
\end{tabular}


Table 1. Countinous

\begin{tabular}{lclll}
\hline \multicolumn{1}{c}{ Species } & Month & Spawning season & Latitude & \multicolumn{1}{c}{ References } \\
\hline Sillaginodes punctata & 3 & Mar-May & $34.15 \mathrm{~S}$ & (Fowler et al., 1999) \\
Sillago bassensis & 7 & Nov-Apr/Sept & $32.03 \mathrm{~S}$ & (Hyndes \& Potter, 1996) \\
Sillago burrus & 3 & Dec-Feb & $32.15 \mathrm{~S}$ & (Hyndes et al., 1996) \\
Sillago robusta & 4 & Dec-Mar & $32.03 \mathrm{~S}$ & (Hyndes \& Potter, 1996) \\
Sillago schomburgkii & 3 & Dec-Feb & $32.15 \mathrm{~S}$ & (Hyndes \& Potter, 1997) \\
Sillago vittata & 3 & Dec-Feb & $32.15 \mathrm{~S}$ & (Hyndes et al., 1996) \\
Solea solea & 3 & Dec-Feb & $44.00 \mathrm{~N}$ & (Amara, R. et al., 1994) \\
Sparus auratus & 2 & Nov-Des & $36.40 \mathrm{~S}$ & (Zeldis \& Francis, 1998) \\
Sparus auratus & 2 & Nov-Des & $36.40 \mathrm{~S}$ & (Zeldis \& Francis, 1998) \\
Spondyliosoma cantharus & 2 & Jan-Feb & $28.00 \mathrm{~N}$ & (Pajuelo \& Lorenzo, 1999) \\
Tenualosa toli & 6 & Mar-Oct & $03.30 \mathrm{~N}$ & (Blaber et al., 1996) \\
Theragra chalcograma & 6 & Feb-Jul & $58.00 \mathrm{~N}$ & (Hinckley, S., 2001) \\
Thunnus albacares & 2 & Jul-Aug & $28.55 \mathrm{~N}$ & (Lang et al., 1994) \\
Trematomus bernacchii & 2 & Oct-Nov & $60.00 \mathrm{~S}$ & (Vacchi et al., 1996) \\
Trematomus bernacchii & 2 & Oct-Nov & $68.30 \mathrm{~S}$ & (Vacchi et al., 1996) \\
Trematomus hansoni & 3 & Dec-Feb & $60.00 \mathrm{~S}$ & (Vacchi et al., 1996) \\
Trematomus hansoni & 3 & Sept-Nov & $68.30 \mathrm{~S}$ & (Vacchi et al., 1996) \\
Trichiurus lepturus & 4 & Mar-Jun & $22.00 \mathrm{~N}$ & (Kwok \& Ni, 1999) \\
Trichiurus lepturus & 4 & Nov-Feb & $30.00 \mathrm{~S}$ & (Martins \& Haimovici, 2000) \\
Trichiurus nanhaiensis & 5 & Apr-Aug & $22.00 \mathrm{~N}$ & (Kwok \& Ni, 1999) \\
Trisopterus esmarki & 2,5 & Mar-May & $58.00 \mathrm{~N}$ & (Albert, 1994) \\
Upeneus moluccensis & 5 & Jul-Oct & $20.00 \mathrm{~N}$ & (Golani, 1994) \\
Upeneus pori & 7 & Apr-Oct & $20.00 \mathrm{~N}$ & (Golani, 1994) \\
Urophycis brasiliensis & 7 & Jul-Des & $34.54 \mathrm{~S}$ & (Acuna et al., 2000) \\
Urophycis brasiliensis & 7 & Jun-Des & $31.30 \mathrm{~S}$ & (Acuna et al., 2000) \\
Urophycis brasiliensis & 3 & Mar-May & $31.30 \mathrm{~S}$ & (Acuna et al., 2000) \\
Urophycis brasiliensis & 3 & Mar-May & $34.40 \mathrm{~S}$ & (Acuna et al., 2000) \\
\hline & & & &
\end{tabular}

Most teleost fishes have been shown to have seasonal reproduction at all latitudes. Some teleosts living at low latitudes do not show a seasonal reproductive patterns. For example the skipjack, Katsuwonus pelamis, $\left(20^{\circ} \mathrm{N}-20^{\circ} \mathrm{S}\right)$ (Stequert \& Ramcharrun, 1996), the dolphin fish, Coryhaena hippurus $L,\left(23^{\circ} \mathrm{N}\right)$ (Wu et al., 2001), and the damselfih, Crypsotera rolandi $\left(5^{\circ} \mathrm{S}\right)$ (Srinivasan et al., 2002) spawn throughout the year. There are also some other species which spawn over a relatively long period, such as the bone fish, Decpterus macrosoma $\left(5^{\circ} \mathrm{N}\right)$ which spawns for 6 months (June to November) (Atmaja, S. B. \& B. Sadhotomo, 2005), and the halfmoon grouper Ephinephelus rivulatus $\left(22^{\circ} \mathrm{S}\right)$ which spawns for 6 months (July to December) (Mackie, 2000 ), both of them living in tropical waters.

Not all species living at low latitudes spawn all year around or have longer spawning periods than those living at high latitudes. Some low latitude species can have a fairly short spawning duration e.g the whale shark, Rhincodon typus, $\left(16.30^{\circ} \mathrm{N}\right)$ and spanish mackerel, Scomberomus commerson, $\left(19^{\circ} \mathrm{S}\right)$ each spawning for 2 months during spring (Mcpherson, 1993).

To overcome this problem I have grouped studies based on six wider latitudinal ranges. These groupings are: 1) the southern polar zone (60 to $\left.90^{\circ} \mathrm{S}\right) ; 2$ ) the northern polar zone $\left(60\right.$ to $\left.\left.90^{\circ} \mathrm{N}\right) ; 3\right)$ the southern temperate zone $\left(30\right.$ to $\left.\left.60^{\circ} \mathrm{S}\right) ; 4\right)$ the northern temperate zone $\left(30\right.$ to $\left.\left.60^{\circ} \mathrm{N}\right) ; 5\right)$ the north tropical zone $\left(15\right.$ to $\left.30^{\circ} \mathrm{N}\right) ; 6$ ) the south tropical zone $\left(15\right.$ to $\left.30^{\circ} \mathrm{S}\right)$; and 7$)$ the equatorial zone $\left(15^{\circ}\right.$ $\mathrm{S}$ to $15^{\circ} \mathrm{N}$ ) (Figure 1). There are smaller differences in temperature throughout the year at low latitudes compared to high latitude. Stable environmental properties are likely to provide suitable conditions for both stable growth and reproduction. The equatorial zone $\left(15^{\circ} \mathrm{S}\right.$ to $\left.15^{\circ}, \mathrm{N}\right)$ has the smallest daily and annual temperature variation, therefore marine teleosts living in this zone will rarely experience extreme temperature fluctuations.

On average marine teleosts living at lower latitudes $\left(<30^{\circ}\right)$ have longer spawning periods than species living at high latitudes $\left(>30^{\circ}\right)$ (Figure 2). 


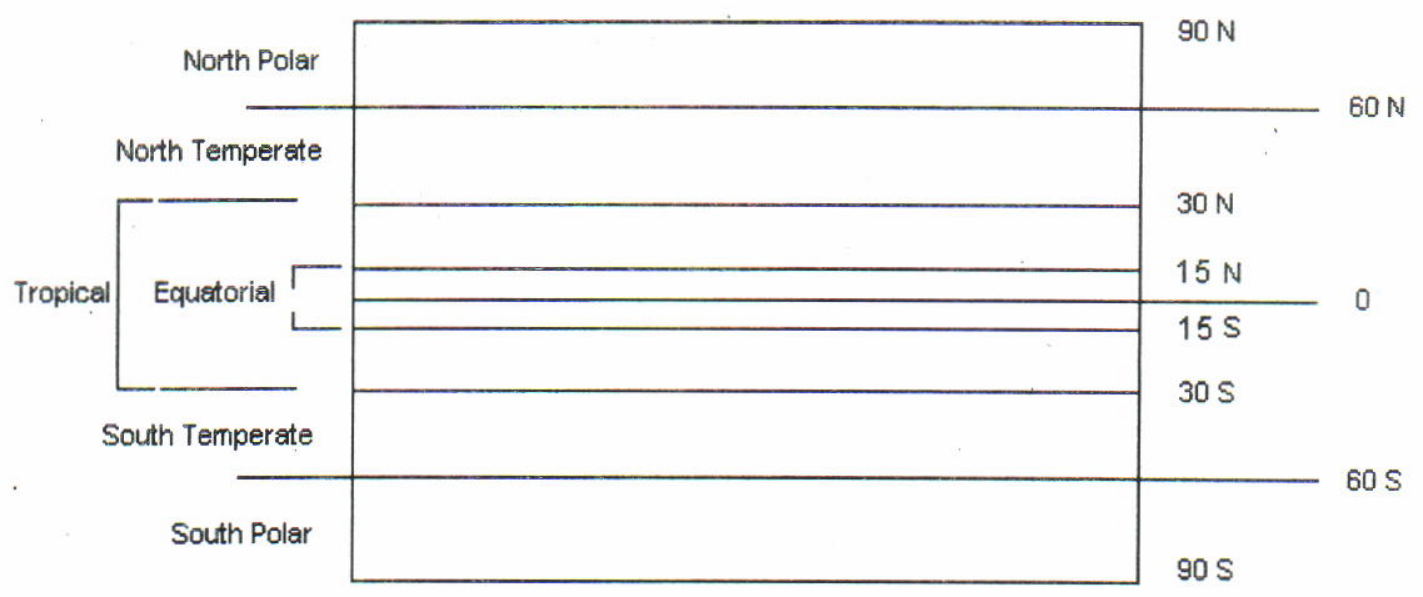

Figure 1. Divisions of Latitudinal range used in this review.

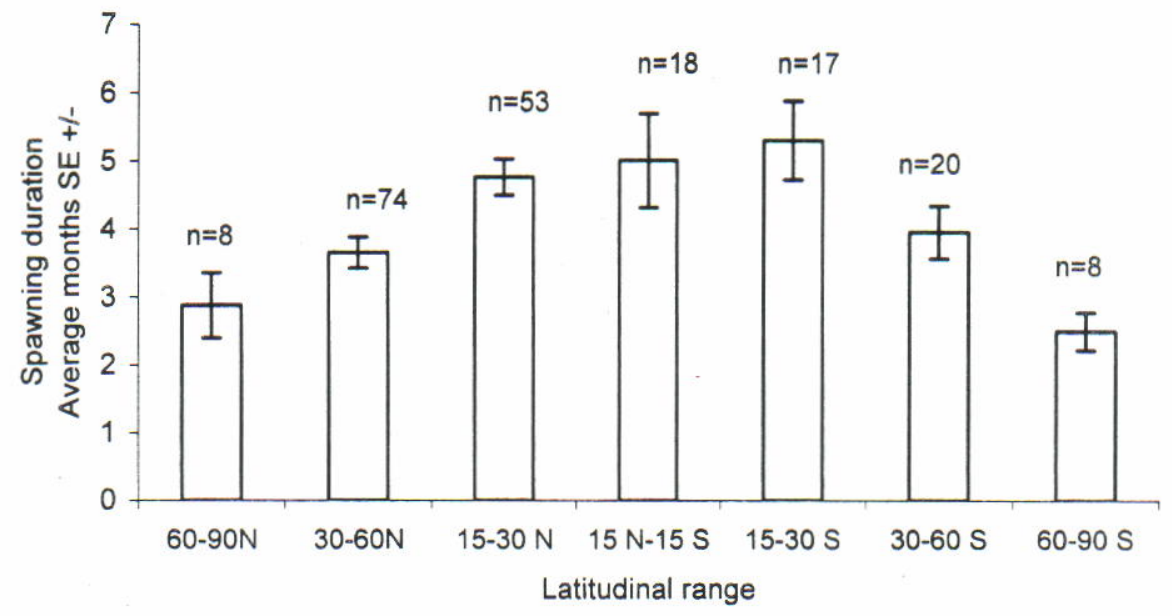

Figure 2. Spawning periods of marine teleosts at different latitudinal ranges (To conform the assumption of using one-way ANOVA test the data were $\log _{10}$ transformed).

There was a trend of increasing spawning duration from polar region $\left(60\right.$ to $\left.90^{\circ} \mathrm{N} / \mathrm{S}\right)$ to the equatorial region $\left(15^{\circ} \mathrm{N}\right.$ to $\left.15^{\circ} \mathrm{S}\right)$, and this trend was significant overall (Figure 2, one way ANOVA, $F=4,878, p=0.000$ ). Spawning duration of marine teleosts living in the equatorial region, however, was not significantly longer than that of species living in the northern and southern tropical zones (15 to $30^{\circ} \mathrm{N}$ and 15 to $30^{\circ} \mathrm{S}$ ) (Table 2, Post hoc comparison, $p=0.570 ; p=0.231$ ). This result is probably due to the small number of studies (15) in the $15^{\circ} \mathrm{N}$ to $15^{\circ} \mathrm{S}$ zone, with only seven of these close to the equator (i.e. $5^{\circ} \mathrm{N}$ to $5^{\circ} \mathrm{S}$ ).

Generally, the spawning durations of species living in low latitudes breed longer than species living in sub tropical regions. Up to 43 out of 73 species in the tropical zone have a spawning duration of 5 to 12 months (Table 1). In the equatorial zone $\left(15^{\circ} \mathrm{N}\right.$ to $\left.15^{\circ} \mathrm{S}\right)$ about 13 out of 18 species have a spawning duration of 5 to 12 months with two species were all year round spawners.

Teleost fishes living both in the northern and southern polar regions (60 to $90 \mathrm{~N} / \mathrm{S}$ ) have significantly lower average of spawning durations than those at lower latitudes. Subtropical teleosts fishes (30 to $60 \mathrm{~N} / \mathrm{S}$ ) also show a significant difference in spawning duration compared to the tropical area $\left(15^{\circ} \mathrm{N}\right.$ to $15^{\circ} \mathrm{S}, 15$ to $30^{\circ} \mathrm{N}$ and 15 to $\left.30^{\circ} \mathrm{S}\right)$. Although the exact time of breeding depends on the species, breeding generally starts later at higher latitudes (Baggerman, 1990). This supports the general assumption that in the tropics, marine teleosts are more likely to have a longer spawning period.

The patterns of spawning duration look closely match the pattern in sea surface temperatures (Figure 3). Water temperature reach a peak of $28^{\circ}$ $\mathrm{C}$ in the tropics and decline below $0^{\circ} \mathrm{C}$ at the polar 
Table 2. Summary of post hoc comparisons of mean spawning duration among the 7 latitudinal ranges (significant value $p<0.005$ ). Only significantly different pairs of latitudinal ranges are shown

\begin{tabular}{ccc}
\hline Latitudinal range & Latitudinal range & p.sig \\
\hline $60-90 \mathrm{~N}$ & $15-30 \mathrm{~N}$ & 0.008 \\
& $15 \mathrm{~N}-15 \mathrm{~S}$ & 0.023 \\
& $15-30 \mathrm{~S}$ & 0.005 \\
$60-90 \mathrm{~S}$ & $15-30 \mathrm{~N}$ & 0.002 \\
& $15 \mathrm{~N}-15 \mathrm{~S}$ & 0.008 \\
& $15-30 \mathrm{~S}$ & 0.002 \\
& $30-60 \mathrm{~S}$ & 0.050 \\
$30-60 \mathrm{~N}$ & $15 \mathrm{~N}-15 \mathrm{~S}$ & 0.024 \\
\hline
\end{tabular}

region. In each of the three main oceans (Indian Ocean, Pacific Ocean and Atlantic Ocean, the temperature increases from the poles towards the equator (Figure 3). As the spawning duration of marine teleosts have longer periods in warmer regions i.e. in the tropics than at the polar region (Figure 2.)

Photoperiod and insolation are the two main factors that determine the temperature regimes of the ocean. Photoperiod regimes differ in their duration in different geographical ranges. The tropical area $\left(30^{\circ} \mathrm{N}\right.$ to $\left.30^{\circ} \mathrm{S}\right)$ has a longer light day ( 8 to $12 \mathrm{~h}$ per day) and is relatively stable within a year. Meanwhile in subtropical areas the daylight is shorter in winter than in summer. The polar regions of the Arctic and Antarctic are the coldest receiving less of the sun's radiation than any other part of the earth's surface.

The sea surface temperatures in the tropic are much more stable than pole regions. For example, the annual temperature variation is much greater at the poles (i.e. $20^{\circ} \mathrm{C}$ ) and in the subtropics (i.e. $25^{\circ} \mathrm{C}$ ) than in tropics (i.e. $5^{\circ} \mathrm{C}$ ) (Bigg, 1996). Temperature regimes are also influenced by seasons showing very different patterns between the equatorial and southern northern hemispheres.
There are three hypothetical temperatures regimes in these three areas regimes. The equatorial zone exhibits a stable cycle through a year, whereas the southern temperate zone will have a temperature peak at about 35 to $40^{\circ} \mathrm{C}$ in December (summer) and decreases to the lowest temperature of $-5^{\circ} \mathrm{C}$ in July (winter). Following the opposite pattern, The northern hemisphere exhibits an opposite pattern reaching the lowest temperature of $-5^{\circ} \mathrm{C}$ in December (winter) followed by an increase to the

\section{PATTERNS OF SPAWNING DURATION RELATED TO FOOD AVAILABILITY}

Food availability may also affect the spawning duration of teleosts fishes. Differences in the timing of spawning in the Cod (Gadus morhua) are not related to temperature but to the timing of plankton production (Brander, 1994). Adequate levels of nutrition must be fulfilled in order to satisfy the animal's physiological condition and ability to complete the reproductive cycle (Nielsen, 1998). The seasonal fluctuations in food availability may determine the timing of reproductive development in some species of teleosts (Collins \& Anderson, 1999). Spawning time has been related to fat content which may reflect the importance of food

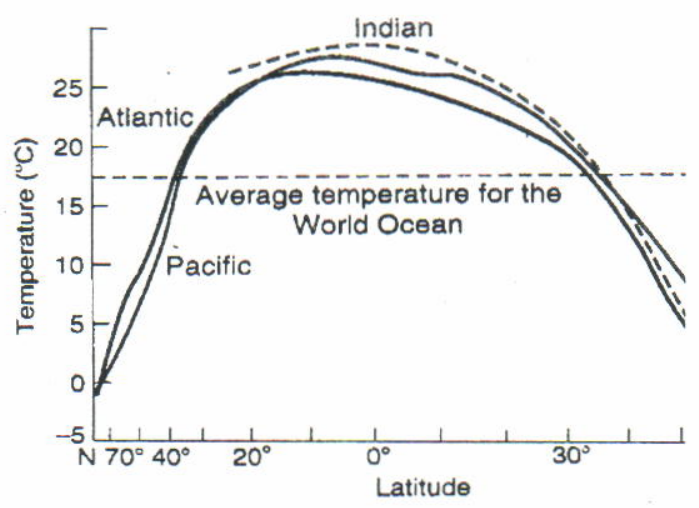

Figure 3. Sea surface temperature in the three major oceans within $70^{\circ} \mathrm{N}$ to $70^{\circ} \mathrm{S}$ (Bigg, 1996). 
maximum value of $40^{\circ} \mathrm{C}$ by July (Summer) (Bigg, 1996) (Figure 4).

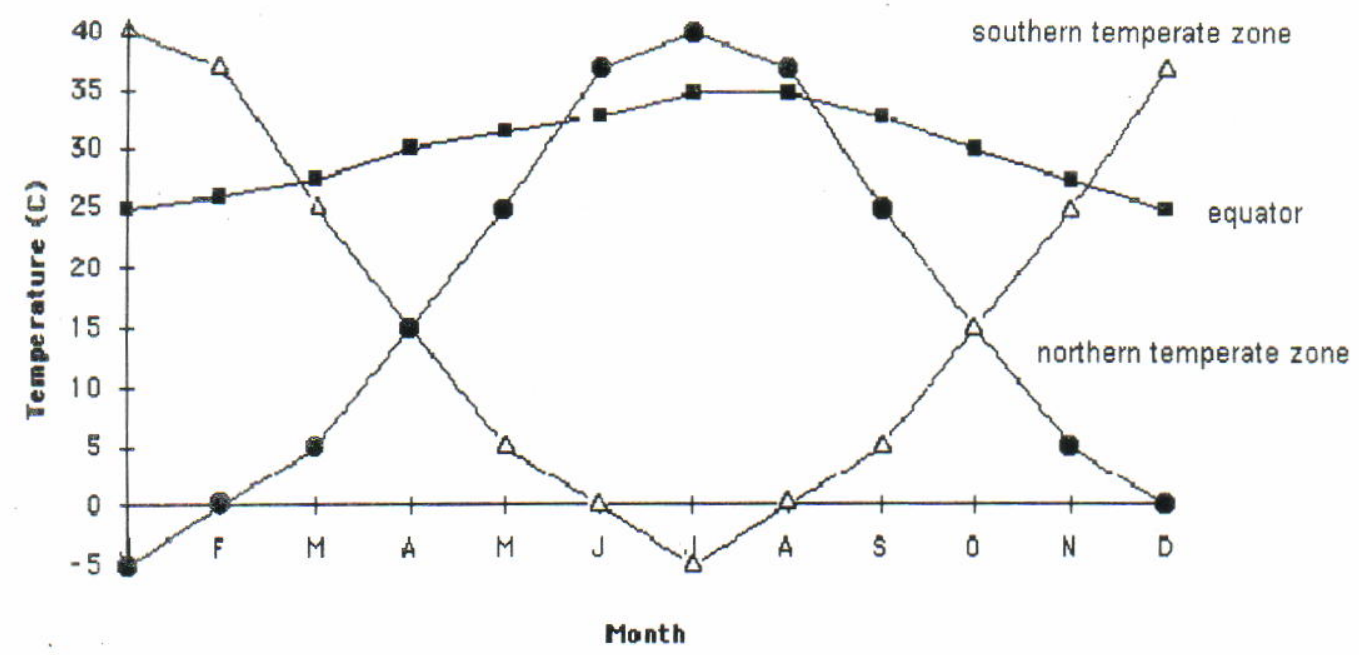

Figure 4. Three hypothetical temperature patterns (equatorial and temperate zones) (Bigg, 1996).

for reproduction. Fish in poor condition (i.e. low fat content) will exhibit delayed maturation (Rajasilta, 1992; Slotte et al., 2000). A restriction on feeding during oocyte recruitment or vitellogenesis, or both, could result in poor condition and thus delay the onset of maturation (Kjesbu et al., 1996), this could also result in fewer maturing females (Bromley et al., 2000) at the start of spawning season.

An increase in food abundance can result in fish storing the maximum energy possible which is accumulated as body fat. This stored energy is to be used for metabolic and reproductive requirements (Paul et al., 1993). A fast increase in body energy/weight indicates that much of the season's energy acquisition takes place in a very short period of intense feeding e.g. $28 \%$ of the Yellowfin sole, Pleuronectes asper, is accumulated within one month during mid May to mid June, when plankton reaches a peak in abundance (Paul et al., 1993). The spring to autumn energy storage strategy is used by the Yellowfin sole and the Northern flat fish species. Inter annual variations in energy storage can, therefore be related to variation in food abundance (e.g. plankton), and could affect the rate of gamete production (Paul et al., 1993).

Phytoplankton and zooplankton, the main food resources of many marine teleosts, are strongly influenced by season (Munro et al., 1990). Thus the seasonality of plankton abundance will affect both growth and reproduction of planktivorous fish.
In the North Atlantic plankton reaches maximum abundance within March to May and decreases to a minimum during November to February (Figure 5). Some fish species in the Northwest Atlantic spawn mainly during periods of high plankton abundance. For example, in three species of butterfly fish Peprilus triachantus, Peprilus burti, Peprilus alepidotus the peak spawning period is March or April (Rotunno \& Cowen, 1997). Similarly, some fish in the northeast Atlantic for examples, the Rosefish, Helicolenus dactylopterus, and the Roundnose Grenadier, Coryphaenoides rupestris, spawn mainly in June which is during the period of peak zooplankton abundance (Allain, 2001).

Plankton abundance in tropical waters is relatively stable throughout the year (Figure 5) (Moyle \& Cech, 1996). Therefore planktivorous fish in this region would rarely experience periods of insufficient food. As a result, teleost fishes will be expected to have stable growth and reproduction: The lack of seasonal variation in food availability. means that tropical teleosts should have long reproductive periods. In contrast plankton abundance in the polar and subtropical regions is more seasonal. This might affect the growth and reproduction of marine teleosts in this region. During periods of high food abundance, marine teleosts in the subtropical and polar regions grow and store energy as fat. During seasons with low food abundance, fish depend on stored energy for their reproduction. 
1. Arctic

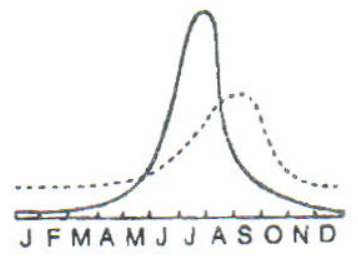

3. North Pacific

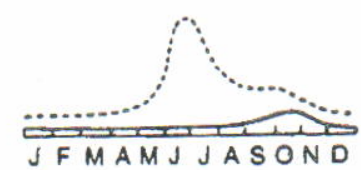

2. North Atlantic

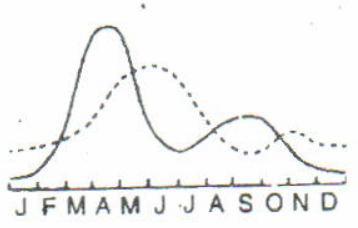

4. Tropical

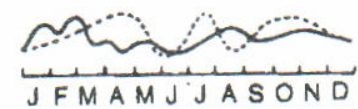

Figure 5. Seasonal productivity in zooplankton and phytoplankton in different parts of the world (Bigg, 1996; Moyle \& Cech, 1996) (dot line = zooplankton; solid line = Phytoplankton).

\section{TIMING OF SPAWNING IN RELATION TO LATITUDE}

There are considerable differences in the timing of spawning among different latitudes. I attempted to look at this variation by relating the peak spawning time (months) from 206 studies of marine fishes among three broad latitudinal ranges (equatorial ( $15 \mathrm{~N}$ to $15 \mathrm{~S}$ ); tropical (15 to $30 \mathrm{~N} / \mathrm{S}$ ) and subtropical (30 to $60 \mathrm{~N} / \mathrm{S}$ ).

A number of trends can be observed from this analysis. The first trend at high latitude, peak spawning periods are generally during spring and summer (i.e. April-July in the northern hemisphere, October to December in the southern hemisphere) (Figure 6). However there are some exceptions, for examples, the Greenland halibut, Reinhardtius hippoglossoides at $73^{\circ} \mathrm{N}$ spawns in DecemberJanuary (Albert et al., 2001); the deepwater squalid shark, Etmopterus princeps at $65.30^{\circ} \mathrm{S}$ spawns in June to July (Jacobsdittir, 2001). The second trend is that teleost fish tend to spawn from January to July (Winte Spring) in the Northern hemisphere within 15 to $30^{\circ} \mathrm{N}$. Fishes within 30 to $60^{\circ} \mathrm{N}$ tend to spawn within June to December (Summer Autumn). Fishes in the south within 15 to $30^{\circ} \mathrm{S}$ are tend to have more peak spawning within October to December (Spring Summer), while those within 30 to $60^{\circ} \mathrm{S}$ fishes tend to reach the peak within January to June (Summer Autumn). A the equator $\left(15^{\circ} \mathrm{N}\right.$ to $\left.15^{\circ} \mathrm{S}\right)$ the peak spawning time pattern is difficult to predict because in this region some teleosts fish seem to show the same spawning intensity all year round.

The timing of the onset of spawning is likely to be related to temperature. Some species of marine teleosts are more likely to start earlier their spawning time or increase their breeding activity in warmer temperatures. 'For example, the red mullet Mullus barbatus and the striped mullet, Mullus surmuletus in the Mediterranean sea, start spawning 4 weeks earlier than their used to due to the warmer temperatures in their habitat (Golani, 1994). Also the dusky grouper, Epinephelus marginatus, at $36^{\circ} \mathrm{N}$ increases it reproductive output five to eightfold during summer (April to June) and decreases it drastically during winter (December to January) (Marino et al., 2001) as the processes of gonadal development are considerably slowed down by low water temperatures $\left(<8^{\circ}\right)$ (Scott, 1990). Meanwhile the spawning of time of the marine plotosid, Cnidoglanis macrocephalus at $28^{\circ} \mathrm{S}$, is related to water temperature with a higher temperature of $22^{\circ}$ $\mathrm{C}$ accelerating the start of spawning by a month compared to the lower temperature of $18^{\circ} \mathrm{C}$ (Laurenson et al., 1993). Spawning in the bluefish Pomatomus saltatrix (L) at around $41^{\circ} \mathrm{N}$ is limited to the warmest months, from July to September when the surface temperature is about $25^{\circ} \mathrm{C}$ (Sabates \& Martin, 1993). Lutjanus Vittus at around $30^{\circ} \mathrm{N}$ spawns for up to 8 months but reaches its peak within the summer time (Cuellar et al., 1996). These examples provide some evidence to support the idea that marine teleosts are likely to begin spawn in the warmer period.

Photoperiod is also widely known as a factor potentially affecting the spawning time, but its effects vary according to species. For example, constant short photoperiod regimes advanced spawning whereas constant long photoperiod period regimes delayed it in the sea bass, Dicentrarchus labrax (Mananos et al., 1997; Prat et 


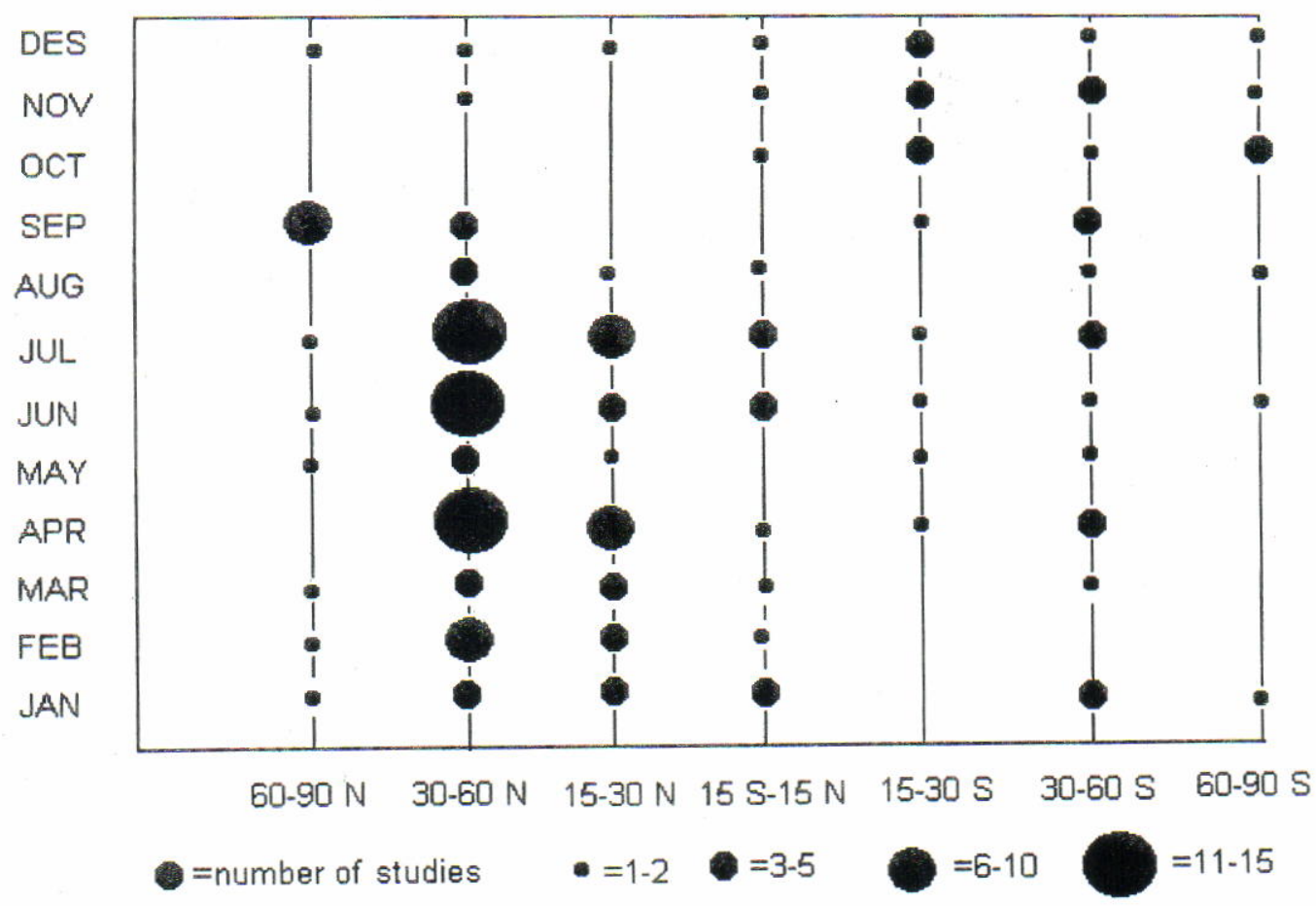

Figure 6. Variability in spawning time within a year in marine teleosts at different latitudes.

Figure 7.

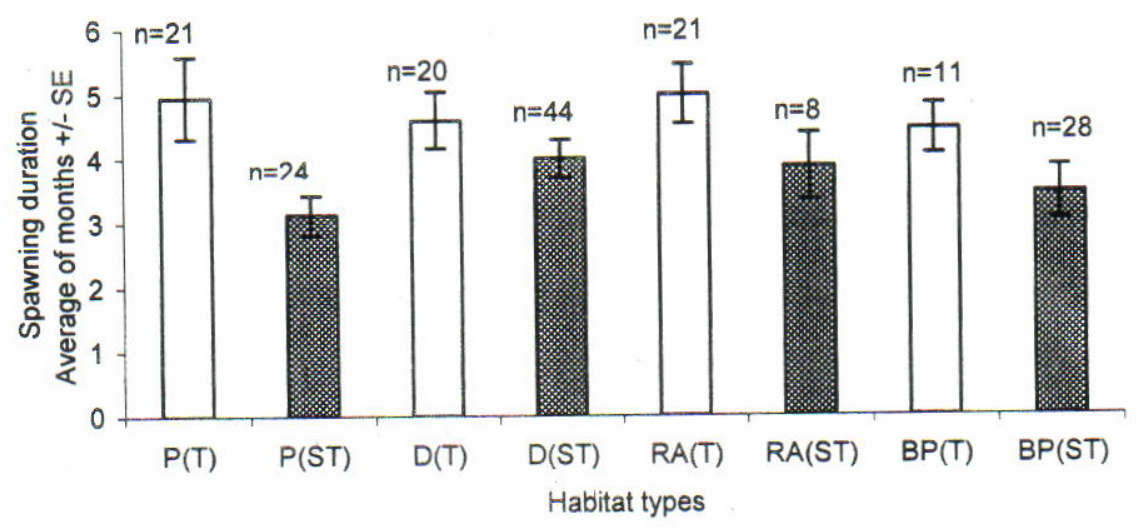

Spawning duration of marine teleosts with respect to habitat between two latitudinal ranges subtropical $\left(30\right.$ to $60^{\circ} \mathrm{S}$ and $\left.\mathrm{N}\right)$, and tropical $\left(30^{\circ} \mathrm{S}-30^{\circ} \mathrm{N}\right)$.

Remarks: $\mathrm{T}=\operatorname{Tropical}\left(30^{\circ} \mathrm{N}-30^{\circ} \mathrm{S}\right) ; \mathrm{ST}=$ Subtropical $\left(30\right.$ to $60^{\circ} \mathrm{N}, 30$ to $\left.60^{\circ} \mathrm{S}\right) ; \mathrm{P}=$ Pelagic; $\mathrm{D}=$ Demersal; $\mathrm{RA}=$ Reef associated; $\mathrm{BP}=$ bathypelagic; To conform to the assumptions of ANOVA, data were $\log _{10}$ transformed

al., 1999). In contrast, studies on salmonids show the opposite pattern with a delay in the start of spawning by constant short photoperiod and an advance by constant long photoperiod (Takashima \& Yamada, 1984). This indicates that photoperiod potentially affects the reproductive timing. However, to validate this assumption more reproductive studies are required to examine the effect of photoperiod on a wider range of species.
DIFFERENCES BETWEEN DEMERSAL AND PELAGIC FISHES IN THEIR PATTERNS OF DURATION OF SPAWNING SEASON

Habitat differences may also be associated with different environmental factors such as temperature, salinity and water pressure as well as food availability. Patterns observed in demersal fish which mostly spend their lifetime close to the sea bed may differ from pelagic fishes which live close 
to the sea surface. Associated seasonal changes in food availability and water temperature can lead to changes in fish behaviour. Pelagic fish biologically are more likely able to respond to changes by moving from place to place. For example The skipjack tuna, katsuwonus pelamis, migrates from the south $\left(20^{\circ} \mathrm{S}\right)$ to the north $\left(20^{\circ} \mathrm{N}\right)$ in order to inhabit areas with conditions suitable for growth and reproduction (Hunter et al., 1986; Stequert \& Ramcharrun, 1996). This result in this species having a relatively long reproductive period. Skipjack tuna spawning occurs throughout the year in tropical waters and seasonally in subtropical waters in all major oceans (Nishikawa et al., 1985). They are oppurtunistic in their reproductive strategy and are thought to spawn throughout their distribution whenever water temperatures rise above $24^{\circ} \mathrm{C}$ (Schaefer, 2000).

Analysis of spawning durations of pelagic and demersal fishes did not support the prediction that pelagic fish have longer spawning durations (Figure 7, Table 3). This may be due to the fact that not all pelagic fishes are able to increase the length of their spawning season by moving to areas where conditions are favourable. It may also be due to a lack of data for pelagic species.

\section{CONCLUSION}

This review confirmed that the timing and duration of spawning of marine teleost fishes is related to latitude. The spawning duration of fishes living in the equatorial region and the tropical regions is generally longer than that of species living in the subtropical and polar regions. In the equatorial peak spawning time is spread throughout the year, whereas at high latitude peak spawning periods were generally during summer. In terms of the spawning duration of marine teleosts with respect to their habitat found that pelagic fish were not different to demersal fishes. The main problem encountered was the paucity of studies on species centred on the equator. More reproductive studies on marine teleosts are still required for equatorial species.

\section{REFERENCES}

Albert, O. T., Nilssen, E. M., Stene, A., Gundersen, A. C., \& Nedreaas, K. H. 2001. Maturity classes and spawning behaviour of Greenland halibut (Reinhardtius hippoglosides). Fisheries Research. 51.

Allain, V. 2001. Reproductive strategies of three deep water benthopelagic fishes from the northeast Atlantic Ocean. Fisheries Research. 51: $165-176$.

Atmaja, S. B. \& B. Sadhotomo. 2005. Study on the reproduction of Layang Deles shortfin scad (Decapterus macrosoma) in the Java Sea. Indonesian Fisheries Research Journal.

Baggerman, B. 1990. Stiklebacks. In Munro A. D., Scott A. P., Lam T. J. (eds) Reproductive Seasonality in Teleosts: Environmental Influences. C. R. C. pess. Florida.

Beddow, T. A., Deary, C., \& Mckinley, R. S. 1998. Migratory and reproductive activity of radio tagged arctic char (Salvelinus alpinus $L$ ) in northern labrador. Hydrobiologia. 372: 249-262.

Bigg, G. R. 1996. The oceans and climate Cambridge University Press.

Brander, K. M. 1994. The location and timing of cod spawning around the British Isles. ICES Journal of Marine Science. 51: 71-89.

Bromley, P. J., Ravier, C., \& Witthames, P. R. 2000. The influence of feeding regime on sexual maturation, fecundity, and atresia in first time spawning turbot. Journal of Fish Biology. 56: 264-278.

Bye, V. J. .1990. Temperate marine teleosts. In Munro A. D., Scott A. P., Lam T. J. (eds) Reproductive Seasonality in Teleosts: Environmental Influences. C. R. C. Press. Florida. pp 125-143.

Collins, L. A. \& Anderson, T. A. 1999. The role of food availability in regulating reproductive developtment in female golden perch. Journal of Fish Biology. 55: 94-104.

Crabtree, R. E. \& Bullock L, H. 1998. Age, Growth, and Reproduction of Black Grouper, Mycteroperca Bonaci, in Florida waters. Fishery Bulletin. 96: 735-753.

Crabtree, R. E., Snodgrass, D., \& Harnden, C. W 1997. Maturation and reproductive seasonality in bonefish, Albula vulpes, from the waters of the Florida Keys. Fishery Bulletin. 95: 456-465.

Cuellar, N., sedberry, G. R., \& Wyanski, D. M. 1996. Reproductive seasonality, maturation, fecundity, and spawning frequency of the vermilon snapper, rhomboplites aurorubens, off the Southeastern United States. Fishery Bulletin. 94: 635-653. 
Fowler, A. J., Mcleay L., \& Short, D. A. 1999. Reproductive mode and spawning information based on gonad analysis for the King George whiting (Percoidei: Sillaginidae) from South Australia. Marine and Freshwater Research. 50: 1-14.

Golani, D. 1994. Niche separation between colonizing and indigenous goatfish (Mullidae) along the Mediterranean coast of Israel. Journal of Fish Biology. 45: 503-513.

Hunter, R. J., B. J. Macewicz, \& J. R. Sibert. 1986. The spawning frequency of skipjack tuna, Katsuwonus pelamis, from the south Pacific. Fishery Bulletin. 84. No.24: 895.

Jacobsdottir, K. B. 2001. Biological aspects of two deep water squalid sharks: Centroscyllium fabricii (Reinhardt, 1825) and Etmopterus princeps (Collet, 1904) in Icelandic waters. Fisheries research. 51: 247-265.

Johnson, A. K., Thomas P., \& Wilson Jr R. R. 1998. Seasonal cycles of gonadal developtment and plasma sex steroids levels in Ephinephelus morio, a protogynous grouper in the eastern Gulf of Mexico. Journal of Fish Biology. 52: 502-518.

Kjesbu, O. S., Kryvi, H., \& Norberg., B. 1996. Oocyte size and structure in relation to blood plasma steroid hormones in individually monitored, spawning Atlantic cod. Journal of Fish Biology. 49: 1197-1215.

Laurenson, L. J. B., Neira, F. J., \& Potter, I. C. 1993. Reproductive biology and larval morphology of the Marine plotosid Cnidoglanis macrocephalus (Teleostei) in a seasonally closed Australian estuary. Hydrobiologia. 268: 179-192.

Mackie, M. 2000. Reproductive biology of the halfmoon grouper, Ephinephelus rivulatus, at Ningaloo Reef, Western Australia. Environmental Biology of Fishes. 57: 363-376.

Mananos, E. L., Zanuy, S., \& Carrillo, M. 1997. Photoperiodic manipulation of the reproductive cycle of sea bass (Dicentrarchus labrax) and their effect on gonadal development and plasma $17_{B}$-estradiol and vitollogenin levels. Fish physiology and biochemistry. 78: 63-80.

Marino, G., Azzuro, E., Massarit, A., Finoia, M. G., \& Mandich, A. 2001. Reproduction in the dusky grouper from the southern Mediterranean. Journal of Fish Biology. 58: 909-927.
McPherson, G. R. 1993. Reproductive biology of the narrow barred spanish mackerel (Scomberomorus commersonl Lacepede, 1800 ) in Queensland Waters. Asian Fisheries Science. 6: 169-182.

Moyle, P. B. \& Cech, J. J. 1996. Fishes an introduction to ichthyology 3rd edition. Prentice Hall. New Jersey.

Munro, A. D., Scott A. P., \& Lam, T. J. 1990. Reproductive seasonality in teleosts: Environmental influences. C. R. C Press. Florida.

Nielsen, K. S. 1998. Animal physiology adaptation and environment 5th edition. Cambridge University press. United Kingdom.

Nurhakim, S. 1993. Some reproduction aspects of ikan banyar (Rastrelliger kanagurta) in the Java Sea. Journal of Marine Fisheries Research. No.81. Balai Penelitian Perikanan Laut. Jakarta.

Prat, F., Zanuy S., Bromage N., \& Carrillo M. 1999. Effects of constant short and long photoperiod regimes on the spawning performance and sex steroid levels of female and male sea bass. Journal of Fish Biology. 54: 125-137.

Rajasilta, M. 1992. Relationships between food, fat, sexual maturation, and spawning time of Baltic herring (Clupea harengus membras) in the Archipelago Sea. Canadian Journal of Fisheries and Aquatic Science. 49: 644-654.

Rotunno, T., \& Cowen R. K. 1997. Temporal and spatial spawning patterns of the Atlantic butterfish, Peprilus triacanthus, in the South and middle Atlantic bights. Fishery Bulletin. 95: 785-799.

Sabates, A., \& Martin P. 1993. Spawning and Distribution of bluefish Pomatomus saltatrix (L) in the northwestern Mediterranean. Journal of Fish Biology. 43: 109-118.

Slotte, A., Johannesen A., \& Kjesbu O. S. 2000. Effects of fish size on spawning time in Norwegian spring spawning herring. Journal of Fish Biology. 56: 295-310.

Srinivasan, M., Jones G. P., \& Satria F. 2002. Reproductive seasonality of $C$. rolandi at Kimbe Bay Papua New Guinea. Unpblished. James Cook University. Australia. 
Stequert, B. \& Ramcharrun B. 1996. Reproduction of Skipjack Tuna (Katsuwonus pelamis) from the western Indian Ocean. Aquatic Living Resources. 9: 235-247.

Takashima, F. \& Yamada Y. 1984. Control of maturation in masu salmon by manipulation of photoperiod. Aquaculture. 43: 243-257.

Taylor, M. H. 1990. Estuarine and intertidal teleosts. In Munro A. D., Scott A. P., \& Lam T. J. (eds) Reproductive Seasonality in Teleosts: Environmental Influences. C. R. C. Press. Florida. pp 109-124.
Widodo, J. 1989. Reproductive biology and harvesting strategy of Indian scad, Decapterus russelli (Ruppell, 1830), (Pisces: carangidae) of the Java Sea. Journal of Marine Fisheries Research. No.53. Balai Penelitian Perikanan Laut. Jakarta.

Wootton, R. J. 1990. Ecology of teleost fishes. Chapman and Hall. New York.

Wu, C. C., Su W. C., \& Kawasaki T. 2001. Reproductive biology of the dolphin fish Corphaena hippurus on the east coast of Taiwan. Fisheires Science. 67: 784-793. 